\title{
Estrategias que complementan la capacitación e-learning en el sector productivo
}

\section{Strategies that complement e-learning training in the productive sector}

\author{
SALINAS-AGUIRRE, María del Consuelo†*, HERNÁNDEZ-CUETO, Jaquelina Lizet, YÁÑEZ-
} FLORES, Sara Margarita y EMILIANO-CASTILLO, Carlos Daniel

Universidad Autónoma de Coahuila, Departamento de Postgrado de la Facultad de Ciencia, Educación y Humanidades, Edificio "N" Campus Saltillo, Saltillo Coahuila, México.

ID $1^{\mathrm{er}}$ Autor: María del Consuelo, Salinas-Aguirre / ORC ID: 0000-0002-6542-1813, Researcher ID Thomson: S92442018, CVU CONACYT ID: 615635

ID $1^{\text {er }}$ Coautor: Jaquelina Lizet, Hernández-Cueto / ORC ID: 0000-0002-3728-7434, Researcher ID Thomson: S-85882018, CVU CONACYT ID: 322702

ID $2^{\text {do }}$ Coautor: Sara Margarita, Yáñez-Flores / ORC ID: 0000-0002-4750-4244, Researcher ID Thomson: S-92312018, CVU CONACYT ID: 352125

ID $3^{\text {er }}$ Coautor: Carlos Daniel, Emiliano-Castillo / CVU CONACYT ID: 1095096

DOI: $10.35429 / J O C S .2020 .22 .7 .31 .37$

Recibido: 25 de Enero, 2020; Aceptado 30 de Junio, 2020

\begin{abstract}
Resumen
Investigación cuantitativa de capacitación en línea de aprendizajes para el desarrollo en el trabajo. La perspectiva es pedagógica centrada en el trabajador con sustento en teorías de desarrollo laboral. Realizada con una muestra al lazar de 326 trabajadores de 5 empresas de Coahuila. El objetivo es la optimización de recursos humanos, técnicos y financieros de la fuerza laboral para una productividad con calidad. La administración para el desarrollo del factor humano es sistematizado con encuestas vía internet. El desarrollo y satisfacción de empleados se manifiesta en el comportamiento organizacional y en la calidad total laboral. La inversión empresarial de altos rendimientos de productividad y satisfacción personal está en capacitación. El objetivo es complementar la capacitación con herramientas administrativas del desarrollo del trabajador. Los subtemas están sustentados en teorías del aprendizaje virtual que contempla las dimensiones centrales del trabajo que motivan las tareas: variedad, identidad, valor, autonomía y retroalimentación; metodologías de aprendizaje en línea. La idea de que la tarea en sí, es clave para la motivación de trabajadores en administración; programación estratégica; prospección de carrera laboral; didácticas para adultos; operación de programas con internet; evaluación del entrenamiento en aulas virtuales; rediseño "e learning" y evaluación de la capacitación a distancia.
\end{abstract}

\begin{abstract}
On-the-job development apprenticeship online training quantitative research. The perspective is pedagogical centered on the worker supported by theories of labor development. Made with a random sample of 326 workers from 5 companies in Coahuila. The objective is the optimization of human, technical and financial resources of the workforce for quality productivity. The administration for the development of the human factor is systematized with surveys via the Internet. The development and satisfaction of employees is manifested in organizational behavior and overall quality of work. The business investment for high productivity and personal satisfaction returns is in training. The objective is to complement the training with administrative tools for worker development. The subtopics are supported by theories of virtual learning that consider the central dimensions of the work that motivate the tasks: variety, identity, value, autonomy and feedback; online learning methodologies. The idea that the task itself is key to motivating administration workers; strategic programming; career prospecting; didactics for adults; operation of programs with internet; evaluation of training in virtual classrooms; redesign "e learning" and evaluation of distance training.
\end{abstract}

Citación: SALINAS-AGUIRRE, María del Consuelo, HERNÁNDEZ-CUETO, Jaquelina Lizet, YÁÑEZ-FLORES, Sara Margarita y EMILIANO-CASTILLO, Carlos Daniel. Estrategias que complementan la capacitación e-learning en el sector productivo. Revista de Sociología Contemporánea. 2020. 7-22:31-37.

\footnotetext{
* Correspondencia al Autor (Correo Electrónico: consuelo.salinas@uadec.edu.mx)

$\dagger$ Investigador contribuyendo como primer autor.
} 


\section{Introducción}

Se analiza brevemente los primeros estudios formales del desarrollo de los trabajadores agrupándose por temas desde el nacimiento de la capacitación formal del trabajo, que históricamente refieren datos de sus primeros usos formales en la capacitación y selección de personal para diferentes funciones bélicas, de los hombres para el ejército desde la segunda guerra mundial. Se continúa con el auge del desarrollo organizacional y temas de inclusión y equidad de géneros de los años setenta, en cuanto a la literatura de capacitación e learning se ha venido desarrollando desde el principio los trabajos de capacitación estaban enfocados a la inclusión de personas con capacidades diferentes como los trabajos de Priante Bretón C.M. (2018). Existen estudios de aprendizaje para desarrollo e inclusión del género femenino al sector productivo y el significado del valor trabajo en diversidad de grupos sociales en el trabajo, Fillipi G.L. Estudios de inserción laboral como un problema de desarrollo de identidad laboral Sanhueza Morales M.A., Muñoz T. y otros. Reinserción social en cárceles, Morales A.M., Muñoz N., Fabrega J. y Welsch G. (2012). En el área de salud física, la capacitación y desarrollo aplicados en rehabilitación física García E. Existen con perspectivas de atención a pacientes, sin orientación al trabajo, García E.I. Como reinserción laboral numerosas tesis de derecho laboral de temas de despido injustificado como la de Benítez Gómez B (2012). La capacitación hacia el estudio de actitudes personales para adaptación social Santana Cárdenas S. Hay muchísimas tesis usando el aprendizaje en el trabajo como sinónimo de capacitación dentro de un paradigma tradicional, Roque Montes de Oca M. Se puede apreciar un enfoque de paradigma organizacional de desarrollo educativo tradicional a los trabajadores. Esta literatura revisada desde los inicios de la capacitación formal en el sector laboral refiere a un enfoque administrativo de orientación humanista centrado en el aprendizaje y desarrollo personal del trabajador.

\section{Marco teórico}

Todas las empresas de bienes y servicios del mundo cuando enfrentan dificultades económicas de operación, por diferentes causas, optan por el ahorro de costos inmediatos como bajar los costos de materia prima o sustituir por otras económicas, enfocan los esfuerzos a eficientar sistemas de producción para lograr resultados rentables a corto plazo, fusiones entre iguales en la industria. Con una perspectiva corta sin objetividad, los administradores de empresas, sacrifican los costos que no tengan impacto en la liquidez de la empresa y no afecten directamente las utilidades a corto plazo. Las estrategias más aplicadas son: ahorrar calidad en materia prima, eficientar la mano de obra, disminuir las contrataciones, despedir personal, emplear trabajadores eventuales no calificados, fusionar dos y tres puestos en uno y desaparecer los gastos intangibles como los cursos de capacitación y desarrollo, ya que el resultado no se percibe de inmediato.

En el siglo XXI el mundo que se ha materializado orientándose al control de la economía, del poder político y los países han formado alianzas comerciales llamados Tratados de libre comercio pactando acuerdos arancelarios y de alianzas socio-económicos. Actualmente la ciencia y tecnología con sus avances en los sistemas de información por internet han transformado la forma de vida del hombre, convirtiéndose en una necesidad las comunicaciones y transacciones de negocios por internet. La crisis económica aunada a la pandemia mundial del COVID 19, ha hecho precipitar el cambio de vida y uso de tecnologías virtuales, en un ambiente competitivo, aumentan la diversidad de opciones de ofertas, las preferencias cambiantes del consumidor se diversifican, la explosión rápida de la información, la necesidad de aprendizajes de virtuales se intensifica y se ha convertido en producto de consumo urgente, las políticas públicas han forzado a las empresas a redireccionar estrategias de sobrevivencia, permanencia y transformaciones a procesos productivos automatizados por computadoras y robótica. 
Las empresas tienen que modificar sus paradigmas de pensamiento y operación a trabajos virtuales remotos desde casa modificar su misión, visión, objetivos y estructuras organizacionales, proceso productivos, forma de comunicarse y comercializar, e incluso rediseñan la organización hacia enfoques virtuales con flexibilidad, rapidez, con un enfoque práctico y a la vez humano, que sea sensibilizado con dolorosas y necesarias transformaciones para responder con rapidez y asertividad a tiempos de crisis económica, competencias internacionales y agresividad de los negocios internacionales actuales. La dinámica acelerada moderna, hace pausas, se reinventa respecto a la importancia hacia activos materiales de una compañía, integran los procesos de calidad, en donde los valores estén orientados hacia el éxito económico, político y consumista de los recursos humanos, ahora opacado por una realidad que les obliga a reconsiderar y actuar en pro de la humanidad. Los trabajadores son multifuncionales, por la falta de oferta laboral, con especialización de habilidades, requiere de experiencia y certificaciones laborales por instituciones calificadas, en donde el manejo de la información con técnicas avanzadas y virtuales son imprescindibles, ya lo escribía Naisbitt J. en sus libros de Macotendencias internacionales desde 1985 "el mundo cambiara de una sociedad industrializada hacia una de la información", que otros autores llaman sociedad del 'conocimiento'. Con entorno de creciente internacionalización, con políticas públicas sustentadas en la intuición, escasez de capital, baja oferta laboral, mayor competencia externa y desaceleración económica, obliga a tener una visión estratégica amplia de gran alcance, con productos y/o servicios de calidad orientados a la demanda de los clientes vía internet. Los sistemas organizacionales ya trasnacionales, pasan de una alta tecnología a una tecnología obligada de procesos productivos computarizados, tienden a estructuras administrativas cada vez más planas, virtuales, pequeñas, sencillas, flexibles y prácticas que invierten en capital humano, optimizando todas sus operaciones con los avances tecnológicos de vanguardia.
Esta investigación en parte tiene su etiología en las dimensiones centrales del trabajo que son la fuente de motivación para los trabajadores de acuerdo al modelo de características del trabajo de Hackman J. R. y Oldham G. R. El e learning para capacitación y desarrollo tiene como antecedentes: objetivos estratégicos, misión y visión del negocio y los factores centrales de mayor relevancia para un trabajador, como son:

1. Variedad y enriquecimiento en el trabajo: realización de diferentes actividades laborales.

2. Identidad con las tareas laborales: identificación con el trabajo a realizar, generar y producir una unidad completa del producto/servicio terminado.

3. Importancia del trabajo: es el significado organizacional del estatus social del puesto respecto a las funciones laborales.

4. Autonomía del trabajador: libertad de acción del trabajador dentro de ciertos estándares programados, para que se sienta autorrealizado.

5. Retroalimentación: conocimiento de la evaluación al desempeño personal y de la apreciación del producto terminado.

\section{Metodología}

Etapas de aplicación del modelo e learning: 1. Aplicación de los resultados de una investigación laboral, no experimental y cuantitativa a 274 trabajadores al azar en u corte transeccional en la recolección de datos, de trabajadores de la Unidad Saltillo de la Universidad Autónoma de Coahuila de una población de 410 sujetos. El análisis fue en tres niveles: descriptivo, correlacional y explicativo con un análisis estadístico factorial multivariado por comunalidades a una probabilidad error del 0.25. 2. Generación del modelo e learning a partir de los resultados obtenidos en la investigación "Habilitación Laboral, Alternativas para el desarrollo humano en el trabajo". Bajo la tesis central de estudiar las funciones y características naturales e importantes para el trabajador. 
3. Aplicación piloto para la validez y confiabilidad, que arrojó un 0.92 con Alpha de Crombach. La aplicación formal se realizó en las plantas: Chrysler Derramadero en Saltillo Coahuila y dos plantas de Royal Bilow, una en Rio Bravo Tamaulipas y otra en McAllen Texas EUA 4. En base a los resultados de los atributos importantes para los trabajadores se implementa un sistema de capacitación sincrónica y asincrónica en la plataforma virtual de la planta que para trabajar en aulas virtuales, laboratorios virtuales, programas interactivos de capacitación $\mathrm{y}$ acuerdos con universidades para grados y/o especializaciones.

\section{Resultados y conclusiones}

El aprendizaje en adultos es disímil al de niños, es deseable que sea personalizado de acuerdo a la edad y nivel de desarrollo del trabajador, así como experiencia laboral. Los adultos en la madurez muestran habilidades basadas en las experiencias de preferencia de tipo intelectual y administrativo, lógicamente las capacidades físicas tenderán a decrecer, así mismo los pensamientos y actividades son repetitivos, con mayor resistencia al cambio tareas laborales. El aprendizaje e learning, dentro del desarrollo de las personas, así como la aplicación de técnicas pedagógicas virtuales para trabajadores, necesariamente tendrá que ir evolucionando y transformándose al mismo tiempo que la pedagogía virtual en el campo laboral, integrando nuevas técnicas en procesos de aprendizaje remotos, acordes a las necesidades actuales.

Conclusiones de la aplicación del modelo de aprendizaje.

Los atributos significativos de aprendizaje que sustentan el paradigma virtual pedagógico aplicado al trabajo son: trabajo en línea interactivo, combinando las metodologías asincrónicas y sincrónicas de acuerdo a las necesidades del trabajador, ser creativo y original en el las tareas, la motivación y resultados están ligadas a investigar y a expectativas personales de autorrealización e inmanencia respecto al trabajo, prefieren aprender e innovar en las funciones laborales, adaptarse transformando el trabajo a un estlo personal, quieren trascender creando, las relaciones afectivas son importantes para innovar y adaptarse laboralmente.
Las habilidades mentales básicas son preferidas a las físicas, para aprender en el campo laboral son: analogías, análisis, síntesis, estímulos propioceptivos, razonar, observar, atención selectiva, ser crítico y analítico, prefieren libertad de construir productos, servicios y procesos laborales. Los atributos relevantes de los resultados con mayor robustez estadística son aquellos que refieren a funciones vitales dentro de la naturaleza humana, habilidades mentales, introyección de representaciones analógicas y actitudes que ayudan a la adaptación y desarrollo personal del ser humano. Probablemente esto se deba a la naturaleza de la muestra que son trabajadores de la universidad y la madurez de las personas, que valora intelectual que lo físico pretende intervenir e innovar trabajando para la adaptación y trascender dejando huella en el trabajo. El manejo de las plataformas y trabajos virtuales es preferido por los jóvenes, pero los adultos tendrán que trabajar en ello, para hacer trabajos desde casa.

Algunos hallazgos significativos son que entre más edad y madurez tiene un trabajador es mayor su introyección y seguridad en sí mismo, menor adaptación a cambios repentinos, y necesidad de ser original y trascender creando, y a la vez sentir que el trabajo es parte de su vida misma o inmanencia laboral.

Propuestas de transformación y cambio en la operación del programa de aprendizaje con tecnología y e learning.

El programa de implementación en metodología administrativa de "cascada", de capacitación y desarrollo de personal se divide en tres niveles consecutivos:

1. Personal de alta dirección. Para ejecutivos, directores, gerentes, jefes, asesores y todo el personal que tenga impacto en la toma de decisiones de una organización. Este nicho de capacitación y desarrollo es el de mayor productividad y rendimiento con altas tecnologías digitales, por el nivel de especialización y estudios. Los ejecutivos lo tienen siempre consigo y en oficina computadoras portátiles, tabletas, celulares inteligentes y relojes de pulsera digitales interactivos, así cargando en ellos el aprendizaje $\mathrm{y}$ consultas necesarias, que son maestros cibernéticos personalizados formales e informales.

SALINAS-AGUIRRE, María del Consuelo, HERNÁNDEZCUETO, Jaquelina Lizet, YÁÑEZ-FLORES, Sara Margarita y EMILIANO-CASTILLO, Carlos Daniel. Estrategias que complementan la capacitación e-learning en el sector productivo. Revista de Sociología Contemporánea. 2020 
En éste caso la tecnología vía "on-line" es útil en juntas, asesorías, consultorías, cursos, especializaciones y grados formales de universidades virtuales. Los ejecutivos tienen un potencial de desarrollo ilimitado con altas tecnologías especializadas y puede tomar e learning a voluntad en hora y lugar preferido, incluso en trayectos o viajes de negocios. La variedad en software en plataformas, dominios, aplicaciones, tutoriales, laboratorios virtuales y simulación de situaciones reales son otro ejemplo importante sobre todo en la toma de decisiones, además de divertir y habilitar al usuario, hacen que el director ensaye sobre supuestos virtuales irreales de ensayo y error que simulen situaciones y prospecciones matemáticas para evitar la quiebra real de la empresa. Afortunadamente esta modalidad e learning, ya es utilizada para aprendizajes en las ciencias de la salud, ya usadas en laboratorios para vacunas.

2. Personal administrativo, técnico de gestión y servicios. Empleados de confianza, secretarias, colaboradores, supervisores, etc. Aquí los recursos tecnológicos y de sistemas computacionales, además de agilizar y eficientar los trabajos de oficina, ahorran costos en material de oficina y tiempo en comunicaciones vía internet. La capacitación e learning, enseña habilidades formales, asesora en tutoriales sobre dudas desde operaciones laborales simples en administración de operaciones, hasta complejas mediante tutoriales, películas, videos, programaciones, etc.

Las aplicaciones tecnológicas se extienden a otras funciones de la dirección de personal como en inducción, selección y prospección de personal con tablas de remplazo potencial por ejemplo. Esta parte media del personal puede superarse con cursos de especialización, diplomados y grados académicos universitarios e learning, el trabajador usa herramientas de trabajo como la computadora como recurso de aprendizaje ilimitado en tutoriales, consultas internet, software interactivo y cursos, diplomados y especializaciones certificadas, que le serán útiles en futuras promociones o estudios formales de grados académicos avalados por universidades virtuales, instituciones de certificación laboral y actualmente en cualquier universidad.

\section{Personal operativo y/o empleados de planta.}

Son los trabajadores de mano de obra en línea, que constituyen la fuerza de trabajo pesada y rutinaria del negocio, así como los servicios de limpieza y mantenimiento de planta.

En éste segmento de mercado laboral la tecnología moderna se aplica con la robótica, automatización y sistemas computacionales a los procesos de producción y a la tecnología de capacitación que implica habilitar instrucciones en línea de producción y/o complejas del trabajo con técnicas sencillas como tutoriales, videos, programadores, etc. La televisión como medio de información en comedores, pasillos y estancias difunde la misión, visión y estrategias de la empresa, los programas de seguridad industrial, las políticas y avisos generales y de emergencia, para mensajes subliminales de pertenencia a la empresa. La tecnología se hace presente en los adiestramientos rutinarios del trabajo, pueden sustituirse operaciones físicas para que el trabajador realice actividades mentales o de otro tipo como los montacargas y ensambles computarizados.

Las Técnicas de aprendizaje e learning son diferentes de acuerdo al objetivo y necesidades de capacitación y desarrollo de los trabajadores, al paradigma ideológico subyacente y las nuevas tecnologías y plataformas digitales realizadas a la medida de cada empresa. Actualmente son usadas: conferencias y juntas virtuales, aprendizajes interactivos sincrónicos en aulas virtuales, capacitación asincrónica con tutoriales, videos, películas, simulación de condiciones reales, laboratorios virtuales, estudio de casos, problemas interactivos, lecturas, estudios individuales, enseñanza programada, vistas virtuales a bibliotecas o base de datos internacionales y cursos, diplomados y grados formales en modalidad e learning.

Evaluación al desempeño e learning del aprendizaje a distancia. La manera más fácil y rápida de verificar si un aprendizaje en el trabajador se ha logrado, es con la ejecución de las tareas y/o con los resultados en la calidad y cantidad de los productos o servicios otorgados. 
Para ello, en Administración de personal se elaboran formatos de evaluación al desempeño con los diferentes criterios a medir según la pertinencia, se incluyen los modelos pedagógicos virtuales, sin olvidarla motivación en las dimensiones centrales que importan a todo trabajador y por supuesto los objetivos a enseñar. Los pasos para la evaluación al desempeño son los siguientes: analizar y seleccionar los criterios $\mathrm{y}$ normas a evaluar; realizar un examen y observación del proceso operativo en forma objetiva, anterior a la capacitación; aplicación del programa e learning de capacitación de personal. Previa verificación de los sistemas y herramientas de internet y tecnologías necesarias para aplicarlo; evaluación teórica de los puntos básicos y necesarios para las funciones del trabajo; observación al desempeño en el trabajo, si se aplican los nuevos aprendizajes; seguimiento de los estándares mínimos de ejecución laboral; retroalimentación al trabajador de ejecución del desempeño con perspectivas a oportunidades de mejora.

El modelo e learning elaborado y aplicado al personal deberá ser sujeto a rediseño cuando menos cada año, para realizar las adecuaciones pertinentes y necesarias integradas a la planeación estratégica del negocio. El programa e learning deberá ser flexible, dinámico y adaptativo ante nuevas circunstancias de contingencia, como las que pasmos ahora. El contenido de la capacitación será personalizado a las necesidades de los trabajadores y la empresa. Además se verificará la obsolescencia de tecnologías y herramientas virtuales empelada en capacitación y desarrollo. Las ventajas de la capacitación e learning: beneficios para la empresa en general.
Mayor rendimiento sobre la inversión a largo plazo; promueve la pertenecía a la organización; enseña la misión, visión, objetivos y estrategias del negocio; ayuda al conocimiento de las funciones laborales; capacita a mayor número de empleados con menos cotos, eleva la moral de la fuerza de trabajo; mejora las relaciones jefe subordinado; es un auxiliar en la planeación estratégica y prospecciones de indicadores a futuro; ayuda en la implementación de políticas dentro de la empresa; informa de las necesidades de los trabajadores; proporciona candidatos a promociones de puestos, mantiene bajos los costos de operación; contribuye a la formación de líderes; evita los gastos de consultas externas; permite detectar, prevenir y resolver conflictos, innovar tecnología, proceso, productos, servicios y constituye una clave indispensable para los trabajadores y desarrollo organizacional.

Las ventajas para los trabajadores: mejora la actitud y satisfacción de los empleados; ayuda a tomar decisiones; a desarrollarse innovando en el trabajo; soluciona problemas interpersonales; permite el logro de metas personales y laborales; ayuda a formar en los empleados un sentido de pertenencia a la compañía; elimina los errores de incompetencia en el trabajo y desperdicio de materiales en operaciones defectuosas; aprenden sobre nuevas tecnología y herramientas virtuales aplicadas al trabajo. La capacitación e learning desarrolla las relaciones humanas en la comunicación personal y de grupos de trabajo; sirve para dar inducción a nuevos empleados, orienta en procedimientos operativos y dudas de trabajador; informa de las disposiciones oficiales; ayuda a implementar nuevas políticas; introyectando la misión de la compañía; promueve la cohesión de los equipos de trabajo; motiva hacia el aprendizaje y mejora la calidad de vida en el trabajo.

Las principales desventajas del aprendizaje e learning son: método no presencial, indirecto de aprendizaje; es proclive a problemas en la comunicación por no ser "cara a cara"; requiere capacitación de personal calificado en métodos didácticos virtuales, manejo tecnologías y sistemas de internet; la inversión en equipo es costosa; loa trabajadores adultos y mayores tienen dificultades en su aprendizaje, además de una actitud negativa a las nuevas tendencias virtuales del mundo actual. 
La tecnología nueva y la inteligencia artificial, no podrá sustituir del todo al trabajador, puesto que él controla los comandos del sistema, los sistemas computacionales son una imitación rudimentaria de las funciones mentales humanas, ellas podrán enriquecer las tareas laborales y la calidad de vida, facilitan trabajos, dan rapidez y exactitud al trabajo, para que cumpla con estándares previamente programados. Desaparecerán los trabajos rutinarios y pesados para el hombre, los operadores de máquinas se transforman en trabajadores electrónicos computarizados, los contadores harán mejor los análisis y prospecciones financieras, el jornalero se convierte en operador, el oficinista en programador, el técnico de laboratorio en ingeniero en sistemas computacionales y el maestro en asesor virtual y guía de conocimientos científico.

Reflexión final. La preexistencia actual de un paradigma dominante en donde se adiestra y educa a los trabajadores para el trabajo, con reforzamientos conductistas, en donde los trabajadores se capacitan de acuerdo a las expectativas de los jefes y resultados de la organización. La empresa se contempla como el medio que estimulará a los trabajadores a adaptarse a nuevas formas de vida para cumplir la misión estratégica planeada en las empresas y una adaptación mejor de la persona a nuevos cambios. EL nuevo paradigma laboral que sustenta el modelo de aprendizaje e learning, tiene una etiología psicopedagógica humanista, retoma las actividades laborales como funciones naturales humanas para satisfacer las necesidades vitales del trabajador. Engloba una nueva forma de aprender, enseñar y adaptarse, el trabajo se constituye en vocación y misión de vida, descubriendo, capacitando y desarrollando habilidades nuevas. Así la empresa se transforma a la par para desarrollar seres humanos.

La capacitación e learning de los trabajadores dentro de su propio campo vital se convierte en su modo de vida y la connotación del trabajo cambiará hacia las funciones laborales en línea, administradas en función del propio enriquecimiento como trabajador para dale un sentido de trascendencia, generativa y autorrealización.

\section{Referencias}

Arias Galicia F. (2000). Editorial Trillas, Administración de Recursos Humanos.

Abbagnano N. actualizado por Fornero G, (2012). Diccionario de filosofía. Editorial Fondo de la cultura económica.

Chruden H. y Sherman A. (2001). Administración de Personal. Maximización del Potencial de Empleados. CECSA.

Hodgetts R. M. y Altman S. (2005). Comportamiento en las Organizaciones. Mc Graw-Hill. México.

Davis, K. (2006). Comportamiento Humano en el Trabajo. Editorial Mc Graw-Hill. México

Mcfarland D. E. (2002). Administración de Personal. Editorial Fondo de la Cultura Económica. México.

Mileno Foros (2020).

https://www.milenio.com/milenio-foros/enmexico-solo-8-de-las-empresas-estan-dirigidaspor-mujeres

Quiñones L. (2020) NOTICIAS ONU, Mirada global de historias humanas.

https://news.un.org/es/story/2020/05/1474002

Robbins S. (2006). Comportamiento Organizacional. Editorial Mc Graw-Hill. México.

Schultz D.P. (2004). Psicología Industrial. Editorial Mc Graw-Hill. México.

Werther W.B. y Davis Keith. (2010). Administración de Recursos Humanos Editorial Mc Graw-Hill. México.

Women Now (2020) 26 agosto. https://www.womennow.es/es/noticia/paisesliderados-por-mujeres-onu/.
SALINAS-AGUIRRE, María del Consuelo, HERNÁNDEZCUETO, Jaquelina Lizet, YÁÑEZ-FLORES, Sara Margarita y EMILIANO-CASTILLO, Carlos Daniel. Estrategias que complementan la capacitación e-learning en el sector productivo. Revista de Sociología Contemporánea. 2020 